\title{
Evaluation of Jinlida Granules in Improvements to Standard-Reaching Rate of Blood Glucose and Type 2 Diabetes Mellitus Symptoms: Results of Secondary Analysis From 12-Week Trial
}

\section{De Jin ( 826901274@qq.com )}

China Academy of Chinese Medical Sciences Guanganmen Hospital

\section{Yuqing Zhang}

China Academy of Chinese Medical Sciences Guanganmen Hospital

\section{Yingying Duan}

China Academy of Chinese Medical Sciences Guanganmen Hospital

\section{Xudong An}

China Academy of Chinese Medical Sciences Guanganmen Hospital

\section{Xiaomin Kang}

Beijing University of Chinese Medicine

Fengmei Lian

China Academy of Chinese Medical Sciences Guanganmen Hospital

\section{Xiaolin Tong}

China Academy of Traditional Chinese Medicine: China Academy of Chinese Medical Sciences

\section{Research Article}

Keywords: Jinlida, Chines medicine, Clinical symptom, Secondary analysis

Posted Date: December 13th, 2021

DOI: https://doi.org/10.21203/rs.3.rs-1146252/v1

License: (c) (i) This work is licensed under a Creative Commons Attribution 4.0 International License. Read Full License 


\section{Abstract \\ Background}

The previous study found that the Jinlida could significantly reduce levels of blood glucose and enhance the lowing-glucose action of metformin. However, the role of the standard-reaching rate of blood glucose and improving clinical symptoms in Jinlida has yet to be studied. We aimed to elaborate on the efficacy of Jinlida in Type 2 diabetics who experience clinical symptoms based on secondary analysis from the 12-week trial.

\section{Methods}

Data were analyzed from a 12-week, randomized, placebo-controlled, study of Jinlida. The standardreaching rate of blood glucose, total symptom score, the efficacy of single symptom, symptom disappearance rate, symptom improvement rate was evaluated. Then, the correlation of the symptom effective rate and $\mathrm{HbA} 1 \mathrm{c}$ was analyzed.

\section{Results}

192 T2DM patients were randomly allocated to receive either Jinlida or a placebo for 12 consecutive weeks. The standard-reaching rate of $\mathrm{HbA} 1 \mathrm{c}(<6.5 \%)$ was $25 \%$ in the treatment group when compared to $14.9 \%$ in the control group. $(P=0.046)$; All the symptom exhibited a change difference, comparing the treatment group to the control group after 12 weeks' intervention. $(P<0.05)$; Hunger, polyuria, dry mouth, sweating and dysphoria in chestpalms-soles symptoms exhibited a change difference in the treatment group, compared to that in the control group. $(P<0.05)$. All the symptom exhibited a significant difference in symptom improvement rate. $(P<0.05)$.

\section{Conclusions}

Jinlida glandule can effectively improve clinical symptoms of type 2 diabetics including thirsty, fatigue, hunger, polyuria, dry mouth, night-sweat, dysphoria in chenstplms-soles and constipation. It can be used as an effective adjuvant treatment for T2DM who experience those symptoms. However, these findings need to be further confirmed by evidence-based medicine.

\section{Background}

Diabetes mellitus (DM) is becoming an increasingly serious global public health issue. According to the International Diabetes Federation (IDF), there was a prevalence of 463 million people in 2019, with a prediction for 693 million by 2045 [1]. Epidemiological investigation exhibited that the estimated prevalence of type 2 diabetes was $10.9 \%$ in China [2]. People living with diabetes are at risk of developing 
several serious and life-threatening complications, leading to an increased need for medical care, reduced quality of life, and undue stress on families. Diabetes and its complications, if not well managed, can lead to frequent hospital admissions and premature death. Despite the emergence of new anti-diabetic drugs, the glycemic control achieved is far from perfect, and some drugs may cause adverse events and increase cardiovascular disease as well as ineffectively improve the clinical symptoms [3-5].

Therefore, there is an urgent need for new drugs that are effective with minimal side effects and favorable hypoglycemic effects as well as relieving clinical symptoms. Traditional Chinese medicine has been applied in the treatment of DM for thousands of years. In recent years, Chinese medicine has made significant progress in modernization and globalization. Jinlida granule has been approved by the China Food and Drug Administration (CFDA) as a treatment for type 2 diabetes with good hypoglycemic effect and fewer adverse reactions [6]. In the previous work, our research team conducted a randomized, placebo-controlled clinical trial to evaluate the efficacy and safety of Jinlida in T2DM with a sample size of 192 type 2 diabetics who received the Jinlida or a placebo basis of using Metformin for 12 weeks. Our results show that $\mathrm{HbA1c}$ was reduced more significantly in the Jinlida group [7-9]. Furthermore, recent meta-analysis and systematic reviews of Jinlida granules showed significant reductions in fasting plasma glucose, 2-hour post-load glucose, and HbA1c [10]. Pharmacological studies showed the Jinlida increased the expression of genes associated with mitochondrial function and fat oxidation (11), regulating the insulin signaling pathway and phosphorylation of c-Jun N-terminal kinase (JNK) and p38 mitogen-activated protein kinase (MAPK)[12]. However, as previously anti-diabetic drugs reported, current studies in Jinlida put more emphasis on the effect of blood glucose levels and its potential mechanisms than largely ignore the role of improving clinical symptom in Type 2 diabetes.

Though the efficacy and safety of Jinlida, as well as its potential action mechanisms, were elucidated, the role of improving clinical symptoms in Jinlida has yet to be studied. Hence in this work, we conducted a secondary analysis based on the previous a randomized controlled trial to investigate the role of the standard-reaching rate of $\mathrm{HbA} 1 \mathrm{c}$ and improving clinical symptom in Jinlida to bridge the gap between hypoglycemic agents and improvement in diabetic clinical symptom.

\section{Methods}

\section{Study Design And Population}

A 12-week randomized controlled trial was designed to evaluate the efficacy of Jinlida as an add-on medication in T2DM. All subjects in both groups also continuously received their metformin without any dose change. The average daily metformin dose of subjects in Jinlida group and the placebo group was $1,210 \mathrm{mg}$ and 1,310 $\mathrm{mg}$, respectively. Treatment group: Jinlida granule was orally given at $9 \mathrm{~g}$ three times per day after meals and metformin was given at $1,210 \mathrm{mg} /$ day. Control group: placebo group was orally given at $9 \mathrm{~g}$ three times per day, and metformin was given at 1,310 $\mathrm{mg} /$ day. Patients with T2DM were eligible as following: a) patients with T2DM who had been receiving metformin over 3 months, but had an $\mathrm{HbA} 1 \mathrm{c} \geq 7.0 \%$ and an FPG 7.0-13.9 $\mathrm{mmol} / \mathrm{L}$ or a $2 \mathrm{hPG} \geq 11.1 \mathrm{mmol} / \mathrm{L}$. b) Patients who had taken 
other glucose-lowering medications or insulin in addition to metformin within the past 3 months were also excluded. c) People with diabetes, complications of severe kidney, liver and cardiovascular disease or mental illness, and gestation or preparation for gestation was also excluded.

\section{Outcomes}

During this 12-week study period, all the subjects were assessed at 0, 4, 8 and 12 weeks. All subjects received a clinical symptom evaluation. The primary endpoints were the change from baseline in the standard-reaching rate of blood glucose including $\mathrm{HbA} 1 \mathrm{c}(\mathrm{HbA} 1 \mathrm{c}<6.5 \%,<7 \%), \mathrm{FBG}(<7 \mathrm{mmol} / \mathrm{l})$ and $2 \mathrm{hPG}$ $(<10 \mathrm{mmol} / \mathrm{l}, 11.1 \mathrm{mmol} / \mathrm{l})$ in the two groups. The secondary endpoints were total symptom score, efficacy of single symptom, symptom disappearance rate and symptom improvement rate. Among, the efficacy of a single symptom was marked as disappearance, improvement and ineffective. Correlation analysis between symptom disappearance rate and improvement of blood glucose were also evaluated.

\section{Statistics Analysis}

The primary analyses were performed according to the intention-to-treat (ITT) principle. The missing data was processed by Last observation carried forward (LOCF). Data were presented as means \pm SD for treatment comparisons of mean changes from baseline to week 12 in total symptom score. Categorical variables were expressed as the efficacy of single symptom, symptom disappearance rate and symptom improvement rate. For between-group comparison, t-test or the Wilcoxon rank-sum test was performed. Student's paired t-tests were performed to compare the changes in symptom score before and after the intervention. Categorical variables were compared with the chi-square test. The level of statistical significance was set at $P<0.05$. SPSS 19.0 software (SPSS Inc., Chicago, IL, USA) was used to analyze all the data. Correlation analysis was performed. The correlation between symptom effective rate and $\mathrm{HbA1c}$ were analyzed through Pearson correlation analysis.

\section{Results}

\section{Patients}

In this trial, a total of 186 participants, assigned to 94 in the placebo group and 92 in the Jinlida group, completed the study. The mean HbA1c (\%), FBG, 2hPG, body weight, and BMI in the Jinlida and placebo groups was no statistically significant difference in the baseline data. Demographic information of patients has been reported previously [10].

\section{Standard-reaching Rate Of Blood Glucose}


The standard-reaching rate of $\mathrm{HbA} 1 \mathrm{c}(<6.5 \%,<7 \%)$ was $25 \%, 34.8 \%$ in the treatment group when compared to $14.9 \%, 23.4 \%$ in the control group, respectively. The standard-reaching rate of FBG $(<7 \mathrm{mmol} / \mathrm{l})$, was $34.8 \%$ in the treatment group when compared to $23.4 \%$ in the control group. The standard-reaching rate of $2 \mathrm{HPG}(<10 \mathrm{mmol} / \mathrm{l},<11.1 \mathrm{mmol} / \mathrm{l})$ was $32.6 \%, 10.6 \%$, when compared to $39.1 \%$, $18.1 \%$, respectively. Standard-reaching rate of blood glucose exhibited a statistically significant difference in standard-reaching rate of $\mathrm{HbA1c}(<6.5 \%)$, and standard-reaching rate of $2 \mathrm{hPG}(<10 \mathrm{mmol} / \mathrm{l}$, $11.1 \mathrm{mmol} / \mathrm{l})$ in the treatment group $(P<0.05)$. Although standard-reaching rate of $\mathrm{HbA} 1 \mathrm{c}<7 \%(P=0.06)$ and standard-reaching rate of FBG $<7.0 \mathrm{mmol} / \mathrm{I}(\mathrm{P}=0.079)$ in the treatment group did not a significant difference in the treatment group, the standard-reaching rate of tendency is markable (Table 1).

Table 1

Standard-reaching outcomes from baseline in patients after receiving a treatment group or control group for 12 weeks.

\begin{tabular}{|llll|}
\hline Standard-reaching outcomes & Treatment group & Placebo group & P value \\
\hline $\mathrm{HbA} 1 \mathrm{c}<6.5 \%$ & $25.0 \%$ & $14.9 \%$ & 0.046 \\
\hline $\mathrm{HbA} 1 \mathrm{c}<7.0 \%$ & $34.8 \%$ & $23.4 \%$ & 0.06 \\
\hline $2 \mathrm{HPG}<10.0 \mathrm{mmol} / \mathrm{I}$ & $32.6 \%$ & $10.6 \%$ & 0.001 \\
\hline $2 \mathrm{HPG}<11.1 \mathrm{mmol} / \mathrm{I}$ & $39.1 \%$ & $18.1 \%$ & 0.000 \\
\hline $\mathrm{FBG}<7.0 \mathrm{mmol} / \mathrm{I}$ & $34.8 \%$ & $23.4 \%$ & 0.079 \\
\hline
\end{tabular}

\section{Symptom Disappearance Rate}

All the symptom disappearance rate has an upward trend. However, only five of nine symptoms exhibited a statistical change difference in the treatment group, including hunger, polyuria, dry mouth, sweating and dysphoria in chestpalms-soles, compared to that in the control group. $(P<0.05$; Figure $1 C, D, E, F$, and $H)$. Especially for sweating symptom, comparing the treatment group to the control group after the intervention, the symptom exhibited a significant difference in the treatment group at just week 4 with a faster rate of clinical improvement than another symptom. ( $P<0.05$; Figure $1 F)$.

\section{Symptom Improvement Rate}

Comparing the treatment group to the control group after 12 weeks' intervention, all the symptom exhibited a statistically significant difference in symptom improvement rate in the treatment group. $(\mathrm{P}<$ 0.05 ; Figure 2). Four of nine symptoms exhibited a significant difference in the treatment group at 8 weeks including thirsty, polyuria, dry mouth and dysphoria in chestpalms-soles, compared to that in the control group. $(P<0.05$; Figure $2 A, D, E$, and $H)$. Sweeting symptom in the treatment group achieved a 
statistically significant difference at week 4. ( $P<0.05$; Figure 2F). Notably, though night-sweat symptom achieved a statistically significant difference at week 4 and week 12, it did not exhibit a significant difference at week 8. $(P<0.05$; Figure $2 G)$.

\section{Total Symptom Score}

Comparison with pre-treatment, total symptom score was statistically significant in both groups in week 4 , week 8 , week 12. $(P<0.05$; Figure $3 \mathrm{~A})$. The mean change in total symptom score from baseline to week 12 was -5.45 score in the treatment group, when compared to -2.38 score in the control group $(P=0.023$; Figure 3B).

\section{Efficiency Of Single Symptom}

Nine kinds of symptoms were assessed by this work including thirsty, fatigue, hunger, polyuria, dry mouth, night-sweat, dysphoria in chenstplms-soles and constipation. Every symptom was evaluated as ineffective, improvement and disappeared. All the symptom exhibited a change difference, comparing the treatment group to the control group after 12 weeks of continuous intervention. $(P<0.05$; Figure 4$)$.

\section{Correlation Of Symptom Improvement And Hba1c}

Pearson correlation was applied to analyze the correlation between symptom effective and HbA1c. No significant correlations were noted between total symptom score and $\mathrm{HbA} 1 \mathrm{c}$, comparing the treatment group to the control group after 12 weeks of continuous intervention. Correlation coefficients are 0.13 and $P$ value is 0.072 . (Figure 5).

\section{Discussion}

To our knowledge, the present study is the largest Chinese medicine as an add-on Metformin randomized clinical trial around the world, to date. The major findings from this trial revealed that for type 2 diabetics with ineffectively managed by metformin monotherapy, the effects of phytomedicine - Jinlida granule add-on Metformin is superior to Metformin monotherapy in improving symptoms, which is one of the most frequently use lowering-glucose agents in clinical practices. The superior hypothesis was validation in the full analysis set population.

The control of Type 2 diabetes mellitus (T2DM) has become a global challenge. For the management of diabetes, there are various oral hypoglycemic drugs (OHDs) that are available worldwide, which is major management for most type 2 diabetics. Of all the OHAs to treat T2DM, metformin is the first-line oral hypoglycemic agent for the treatment of type 2 diabetes and recommended by the American Diabetes Association and the European Association for the Study of Diabetes [13]. However, like many loweringglucose agents, metformin may not improve clinical symptoms despite its favorable lowering-glucose 
effects. The treatment strategy is inadequate. The evidence was mainly based on one study. One study was designed as a randomized controlled trial with a dose of Chinese medicine (TM81) or placebo in T2DM, which showed the effect of anti-diabetes and improving clinical symptoms [14]. The necessity to develop new strategies for diabetic patients are at a high demand.

From the current evidence of the trials, both Jinlida and metformin reduced levels of blood glucose, and the curative effect of Jinlida add-on metformin was superior to metformin monotherapy [6-10]. In terms of standard-reaching rate of blood glucose, Jinlida add-on metformin has obvious advantages in comparison to metformin monotherapy. After 12 weeks of treatment, standard-reaching rate of $\mathrm{HbA} 1 \mathrm{c}$ $<6.5 \%, 2 \mathrm{HPG}<10 \mathrm{mmol} / \mathrm{l}, 2 \mathrm{HPG}<11.1 \mathrm{mmol} / \mathrm{l}$ achieved a statistically significant difference. The results indicated that Jinlida add-on metformin might contribute to improve standard-reaching rate of blood glucose. Notably, $\mathrm{HbA} 1 \mathrm{c}<7 \%$ and $\mathrm{FBG}<7 \mathrm{mmol} / \mathrm{I}$ did not show a statistically significant difference, but exhibit a standard-reaching tendency. The non-significant finding might be related to sample size. Due to the small sample size, subgroup analyses were not fully analyzed. In the improving clinical symptoms, after 12 weeks of treatment, the mean decrease from baseline in total symptoms score was -5.45 and -2.38 in the treatment group and control group, respectively. Furthermore, all the single symptoms including thirsty, fatigue, hunger, polyuria, dry mouth, night-sweat, dysphoria in chenstplms-soles and constipation achieved a statistically significant difference. These findings remained consistent in this study [14]. In terms of eliminating symptoms, all the symptoms disappear rate was constantly improved. There were five symptoms of achieving a statistically significant difference. Among that, sweating symptom was improved with a statistically significant difference at week 4 . Findings indicated that Jinlida was the most effective procedure with the best outcomes in sweating symptoms. In consideration of improvement in symptoms rate, we found that all the improvement in symptom rate between two groups manifested statistical significance. Five symptoms achieved a significant difference in improvement in symptoms rate at week 8 . However, the sweating and night-sweat achieved a significant difference at week 4 and week 12 . Subsequently, night-sweat did not show the significant difference at week 8, suggesting that Jinlida has a slower but stable effect, and this is consistent with the general regularity of phytomedicine. Moreover, we analyzed that the correlation between $\mathrm{HbA} 1 \mathrm{c}$ and symptom effective rate, with no significant difference. Thus, its action might be considered to be independent of regulating blood glucose. Findings from this work indicated that Jinlida add-on metformin could effectively improve standard-reaching rate of blood glucose and clinical symptoms than metformin.

Chinese medicine has been widely used in treating T2DM, but there is still a lack of high-qualified clinical evidence. Jinlida is a CFDA-approved Chinese medicine for T2DM interventions [6]. There are nine constituents of Jinlida, namely danshensu sodium salt, puerarin, salvianolic acid B, epimedin B, epimedin $\mathrm{C}$, Icariin, and ginsenosides Rb1, Rc, and Rb2. Several studies have revealed its potential mechanisms; For example, Jinlida can reduce intracellular lipid accumulation and enhance autophagy in NIT-1 pancreatic $\beta$-cells [15] as well as increased expression of genes involved in mitochondrial function and fat oxidation [16]. All the above pre-clinical evidence suggests the potential mechanism of Jinlida. 
These results revealed the efficacy of Jinlida for improving clinical symptoms in diabetes to bridge the gap between hypoglycemic agents and improvement in diabetic clinical symptom. Jinlida can be used to treat patients who experience the clinical symptoms of T2DM. There were also some limitations in the present study. First, subjectivity in assessing subjective symptoms is notable confounding factors. Second, due to the long follow-up interval, the patient's symptoms may not be truly feedback, and this may exist gender differences in the way patients cope with symptoms. Third, the long-term benefits of the Jinlida in clinical improvement in clinical symptoms remains unclear based on the findings of the 12week observation. Future research should be focused on developing more effective strategies for evaluating subjective clinical symptoms and longer follow-up to assess the improvement in clinical symptoms for T2DM.

\section{Conclusion}

Jinlida glandule can effectively improve standard-reaching rate of blood glucose, clinical symptoms of type 2 diabetics, including thirsty, fatigue, hunger, polyuria, dry mouth, night-sweat, dysphoria in chenstplms-soles and constipation, and its action might be independent of regulating blood glucose. It can be used as an effective adjuvant treatment for T2DM who experience those symptoms. However, these findings need to be further confirmed by evidence-based medicine.

\section{Abbreviations}

DM (Diabetes mellitus)

T2DM (Type 2 diabetes mellitus)

IDF (International Diabetes Federation)

CFDA (the China Food and Drug Administration)

JNK (N-terminal kinase)

MAPK (p38 mitogen-activated protein kinase)

ITT (the intention-to-treat)

LOCF (Last observation carried forward)

OHDs (oral hypoglycemic drugs)

\section{Declarations}

\section{Funding statements}


Innovation Team and Talents Cultivation Program of National Administration of Traditional Chinese Medicine. (No: ZYYCXTD-D-202001).

\section{The Authors' Contributions}

De Jin and Yuqing Zhang wrote the manuscript; Yingying Duan, Xuedong An and Xiaomin Kang, performed statistical analysis; Fengmei Lian and Xiaolin Tong designed the protocol and revised the paper.

\section{Declaration of Competing interests}

The authors declare no competing interests.

\section{Acknowledgements}

The authors thank the families and residents who participated in the study for their time and contributions.

\section{Availability of data and materials}

The data sets used in this study may be obtained from the corresponding authors by email by request.

\section{Consent to publish}

The authors have read the journal's authorship agreement and the manuscript has been reviewed by and approved by the authors.

\section{Ethical Approval}

This ethical review was not required.

\section{References}

1. International Diabetes Federation. IDF Diabetes Atlas, 9th edit. Brussels, Belgium: 2019. Available at: https://www.diabetesatlas.org

2. Wang $L, G a o P$, Zhang $M$, et al. Prevalence and ethnic pattern of diabetes and prediabetes in China in 2013. JAMA. 2017; 317:2515-2517. 
3. Nissen SE, Wolski K. Rosiglitazone revisited: An updated meta-analysis of risk for myocardial infarction and cardiovascular mortality. Arch Intern Med. 2010; 170:1191-1201. doi:

10.1001/archinternmed.2010.207

4. Loke YK, Kwok CS, Singh S. Comparative cardiovascular effects of thiazolidinediones: Systematic review and meta-analysis of observational studies. BMJ. 2011;342: d1309. doi:

10.1136/bmj.d1309PMC free articled

5. UK Prospective Diabetes Study (UKPDS) Group, corp-author. Intensive blood-glucose control with sulphonylureas or insulin compared with conventional treatment and risk of complications in patients with type 2 diabetes (UKPDS33) Lancet. 1998; 352:837-853. doi: 10.1016/S01406736(98)07019-6

6. Lian F, Tian J, Chen X, et al. The efficacy and safety of Chinese herbal medicine Jinlida as add-on medication in type 2 diabetes patients ineffectively managed by metformin monotherapy: a doubleblind, randomized, placebo-controlled, multicenter trial[J]. PLoS One, 2015, 10(6): e0130550.

7. Jia W, Weng J, Zhu D, et al. Standards of medical care for type 2 diabetes in China 2019[J]. Diabetes/metabolism research and reviews, 2019, 35(6): e3158.

8. Tian J, Lian F, Yang L, et al. Evaluation of the Chinese herbal medicine Jinlida in type 2 diabetes patients based on stratification: Results of subgroup analysis from a 12-week trial[J]. Journal of diabetes, 2018, 10(2): 112.

9. Zhang Y, Wang W, Ning G. Study on the efficacy and safety of Jinlida in patients with inadequately controlled type-2 diabetes and dyslipidemia under lifestyle intervention (ENJOY LIFE Study) [J]. Journal of diabetes, 2015, 7(2): 268.

10. 10. Lian F, Jin D, Bao Q, et al. Effectiveness of traditional Chinese medicine Jinlida granules as an add-on therapy for type 2 diabetes: A system review and meta-analysis of randomized controlled trials[J]. Journal of diabetes, 2019, 11(7): 540-551.

11. Zang SS, Liu YX, Song GY, et al. Effects of Jinlida granules on lipid accumulation in skeletal muscle and mitochondrial function in insulin resistant rats. Chin Tradit Patent Med. 2014; 36:1371-1376. [in Chinese].

12. Muoio DM, Newgard CB. Mechanisms of disease: molecular and metabolic mechanisms of insulin resistance and beta-cell failure in type 2 diabetes. Nat Rev Mol Cell Biol. 2008; 9:193-205.

13. American Diabetes Association. Standards of medical care in diabetes - 2013. Diabetes Care. 2013;36(Suppl 1): S11-S66. doi: 10.2337/dc13-S011.

14. Tong X L, Wu S T, Lian F M, et al. The safety and effectiveness of TM81, a Chinese herbal medicine, in the treatment of type 2 diabetes: a randomized double-blind placebo-controlled trial[J]. Diabetes, Obesity and Metabolism, 2013, 15(5): 448-454.

15. Wang D, Tian M, Qi Y, et al. Jinlida granule inhibits palmitic acid induced- intracellular lipid accumulation and enhances autophagy in NIT-1 pancreatic $\beta$ cells through AMPK activation. J Ethnopharmacol. 2015;161:99-107. 
16. Zang SS, Liu YX, Song GY, et al. Effects of Jinlida granules on lipid accumulation in skeletal muscle and mitochondrial function in insulin-resistant rats. Chin Tradit Patent Med. 2014; 36:1371-1376. [in Chinese].

\section{Figures}

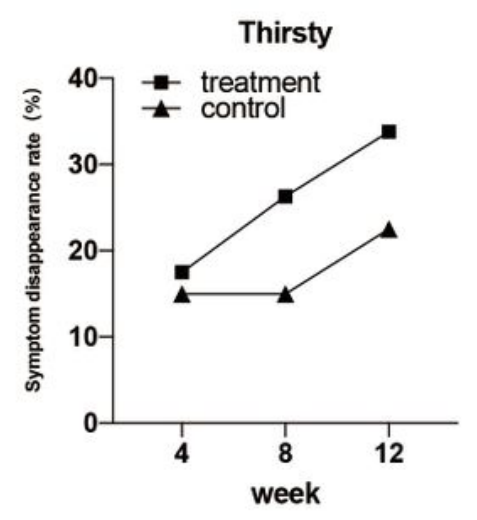

A

Polyuria

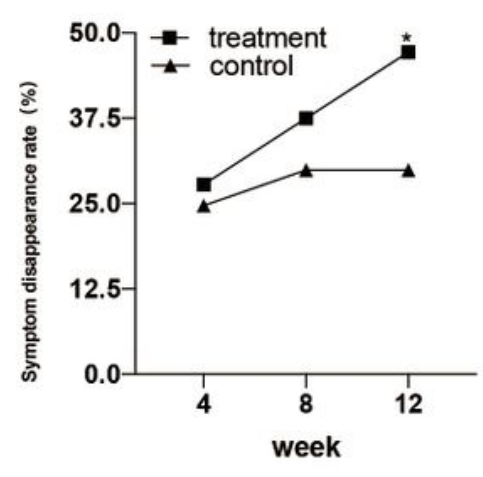

D

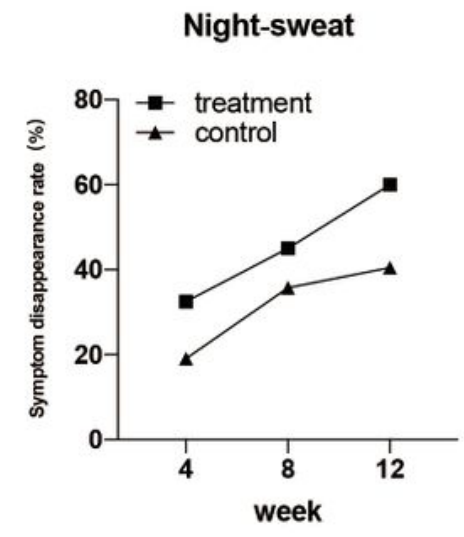

G
Fatigue

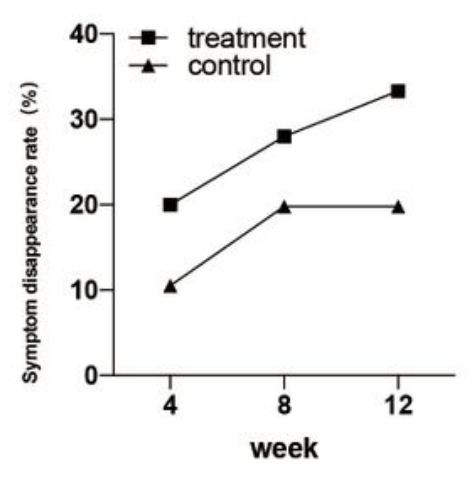

B

Dry mouth

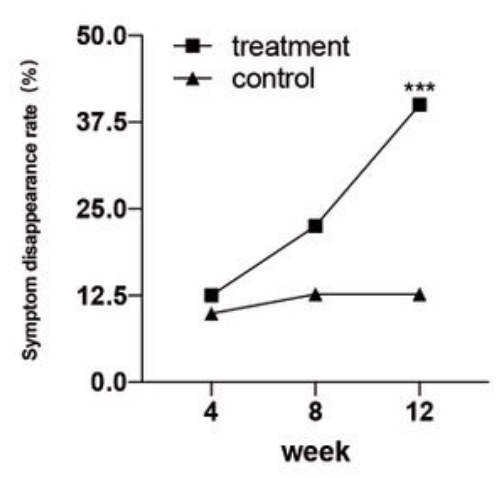

E

Dysphoria in chestpalms-soles

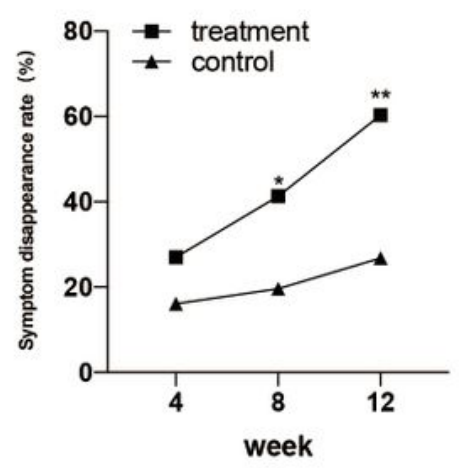

H

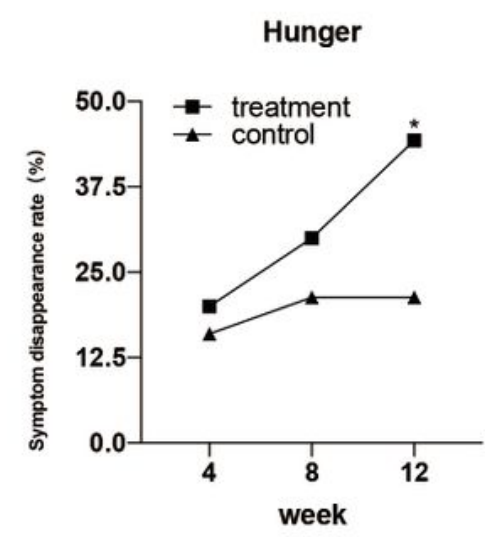

C

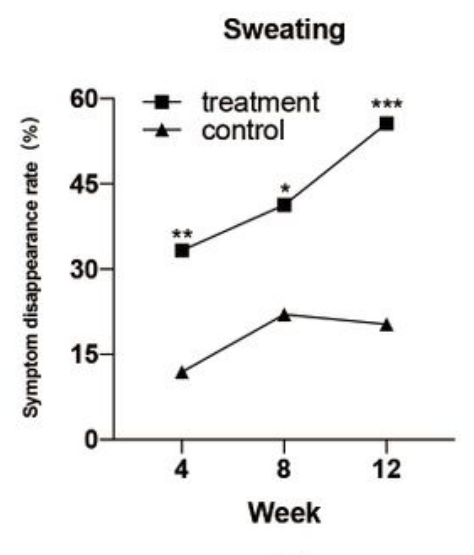

F

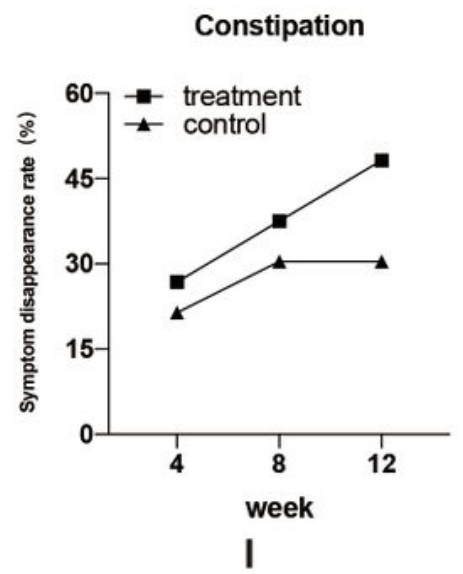


Change in symptom disappear rate from baseline in patients after receiving a treatment group or control group for 12 weeks. Data are from all 192 patients in the full analysis set and are expressed as mean (bar show standard error of the mean). ${ }^{*}$ Compare of outcome between group $P<0.05,{ }^{\star *} P<0.01,{ }^{\star \star \star}{ }^{*} P<0.001$; \# compare of baseline outcome within group $\mathrm{P}<0.001$.

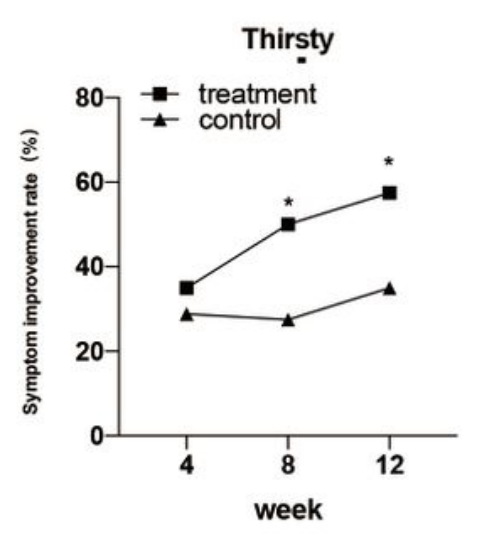

A

Polyuria

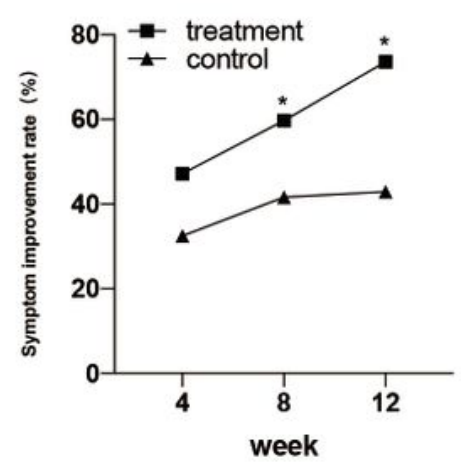

D

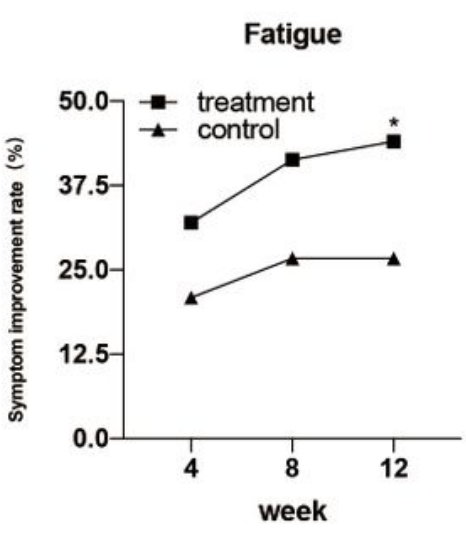

B

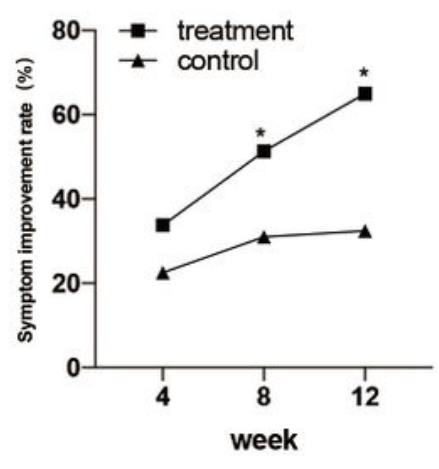

E

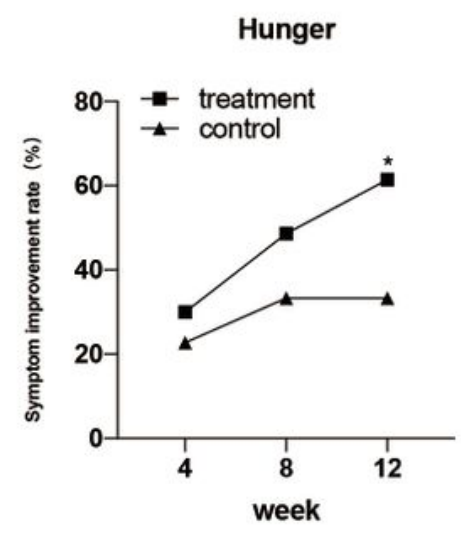

C

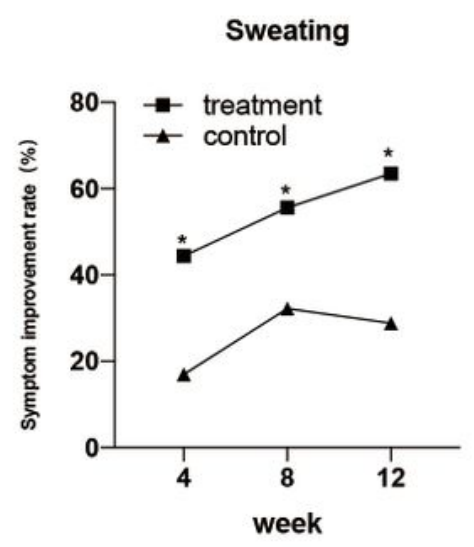

$\mathrm{F}$
Night-sweat

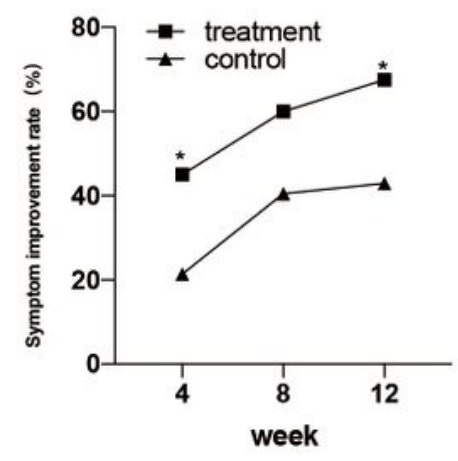

G
Dysphoria in chestpalms-soles

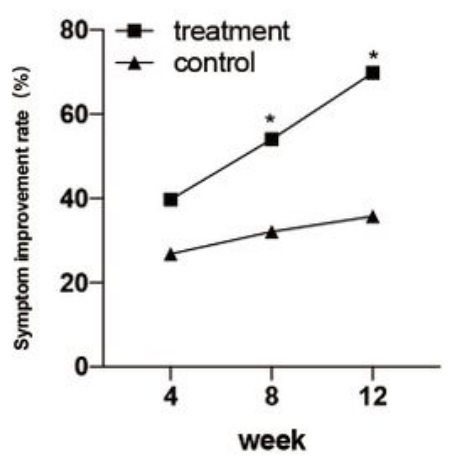

$\mathrm{H}$

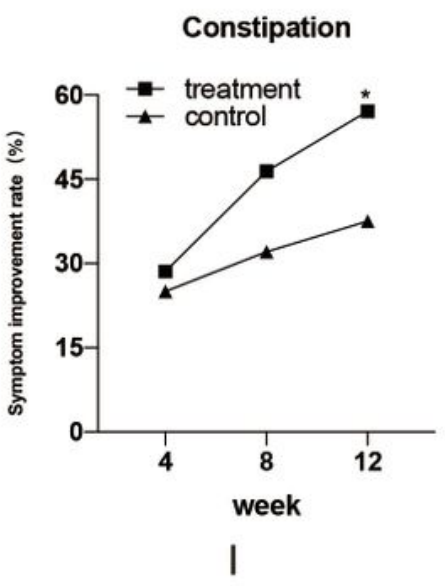

Figure 2 
Change in symptom improvement rate from baseline in patients after receiving a treatment group or control group for 12 weeks.
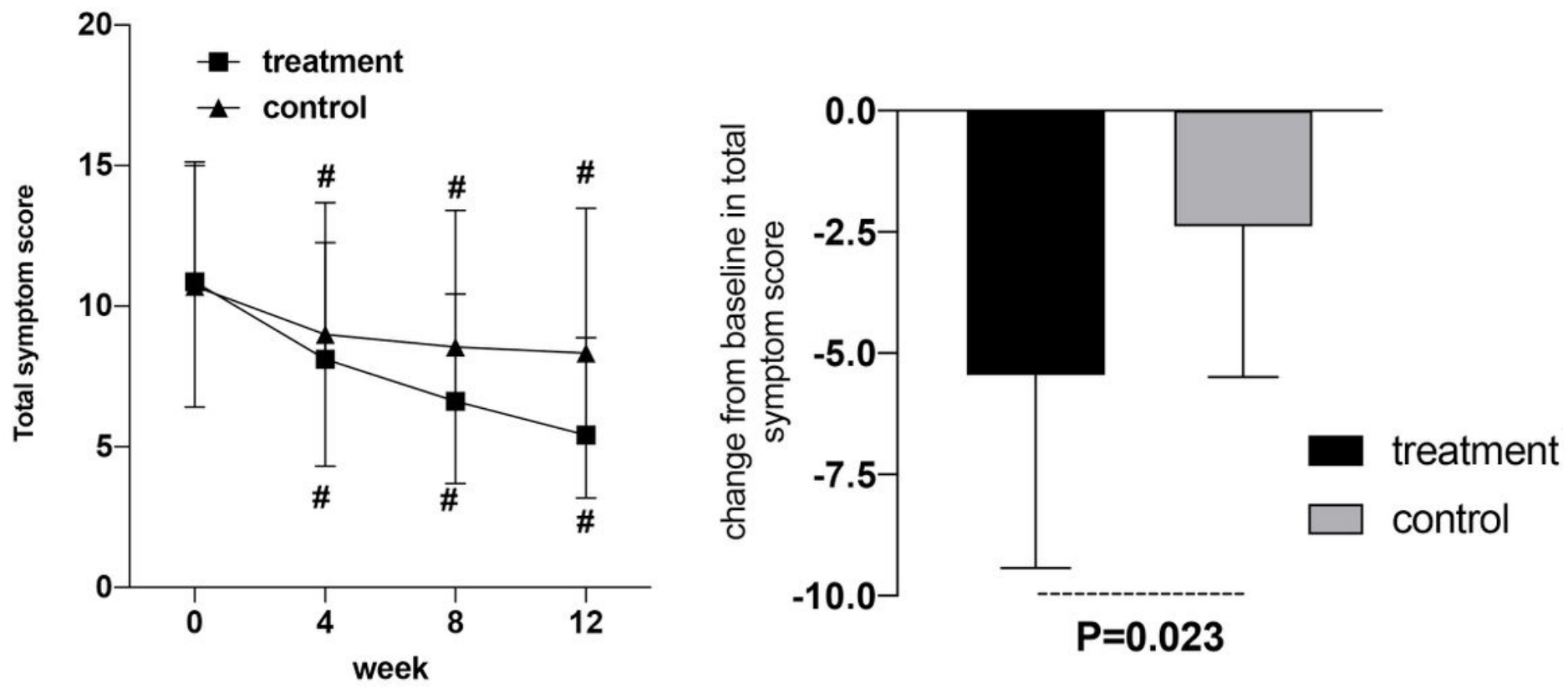

Figure 3

Changes in total symptoms score from baseline in the two group. 


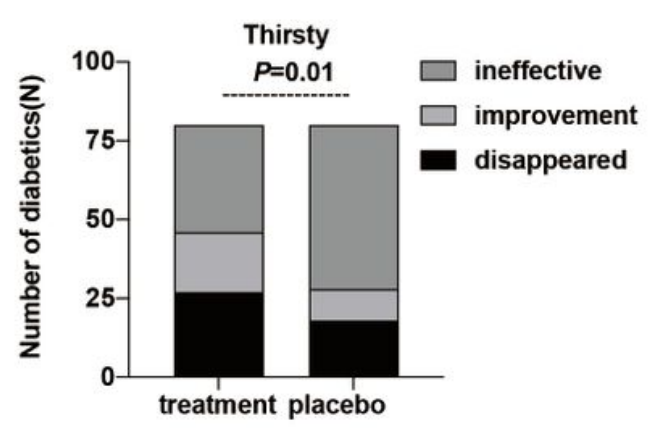

A

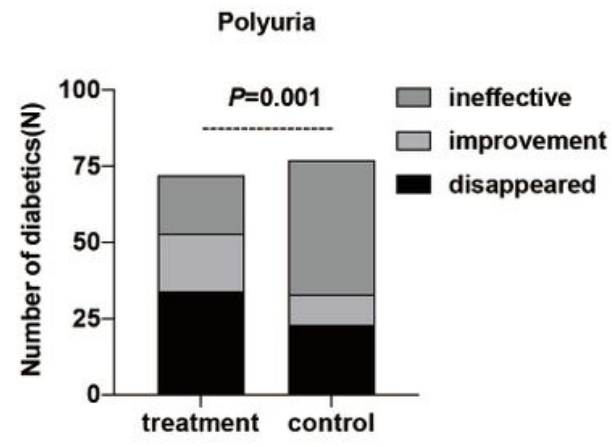

D

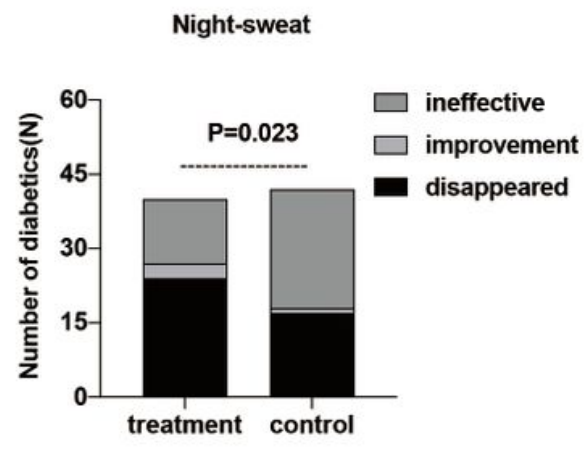

G

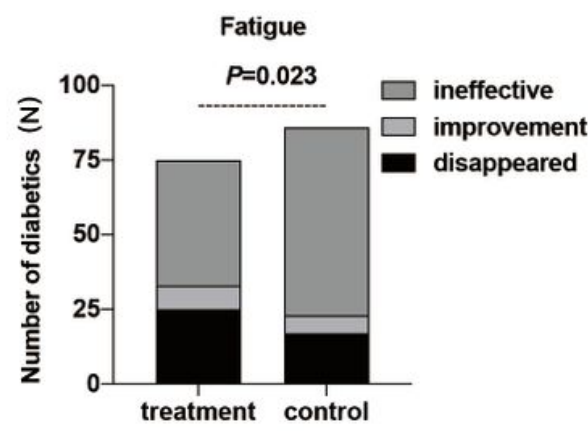

B

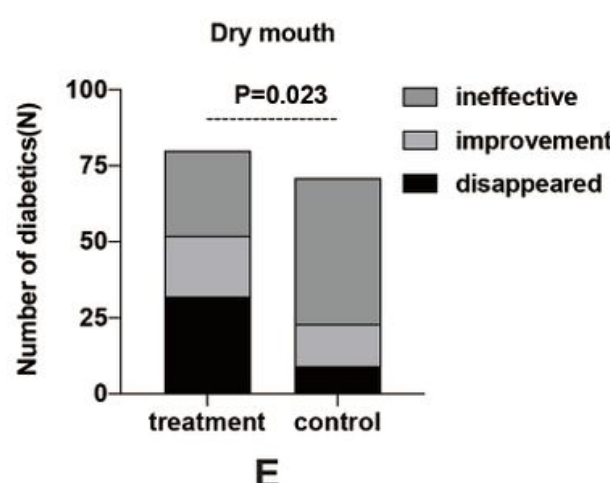

E

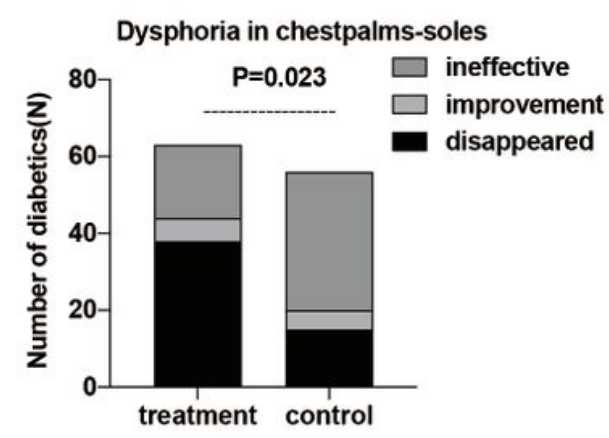

$\mathrm{H}$

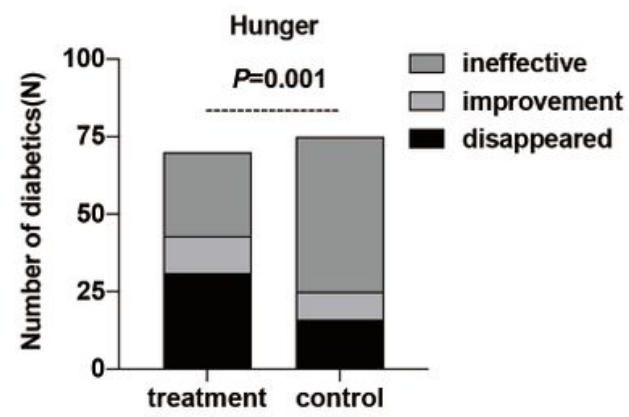

C
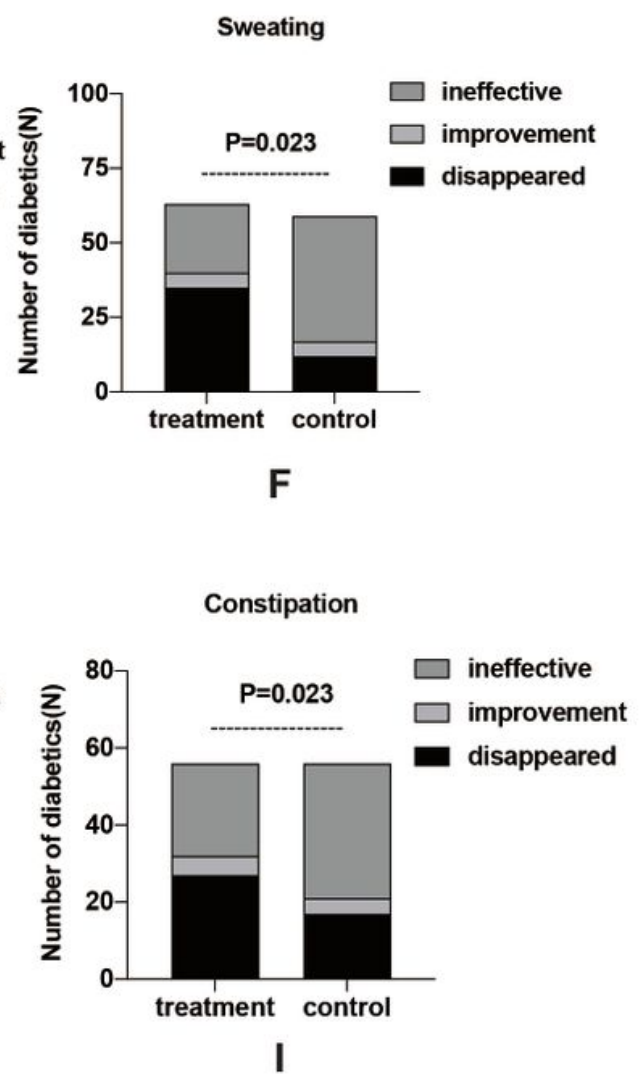

\section{Figure 4}

Change in single symptoms from baseline in patients after receiving a treatment group or control group for 12 weeks. 


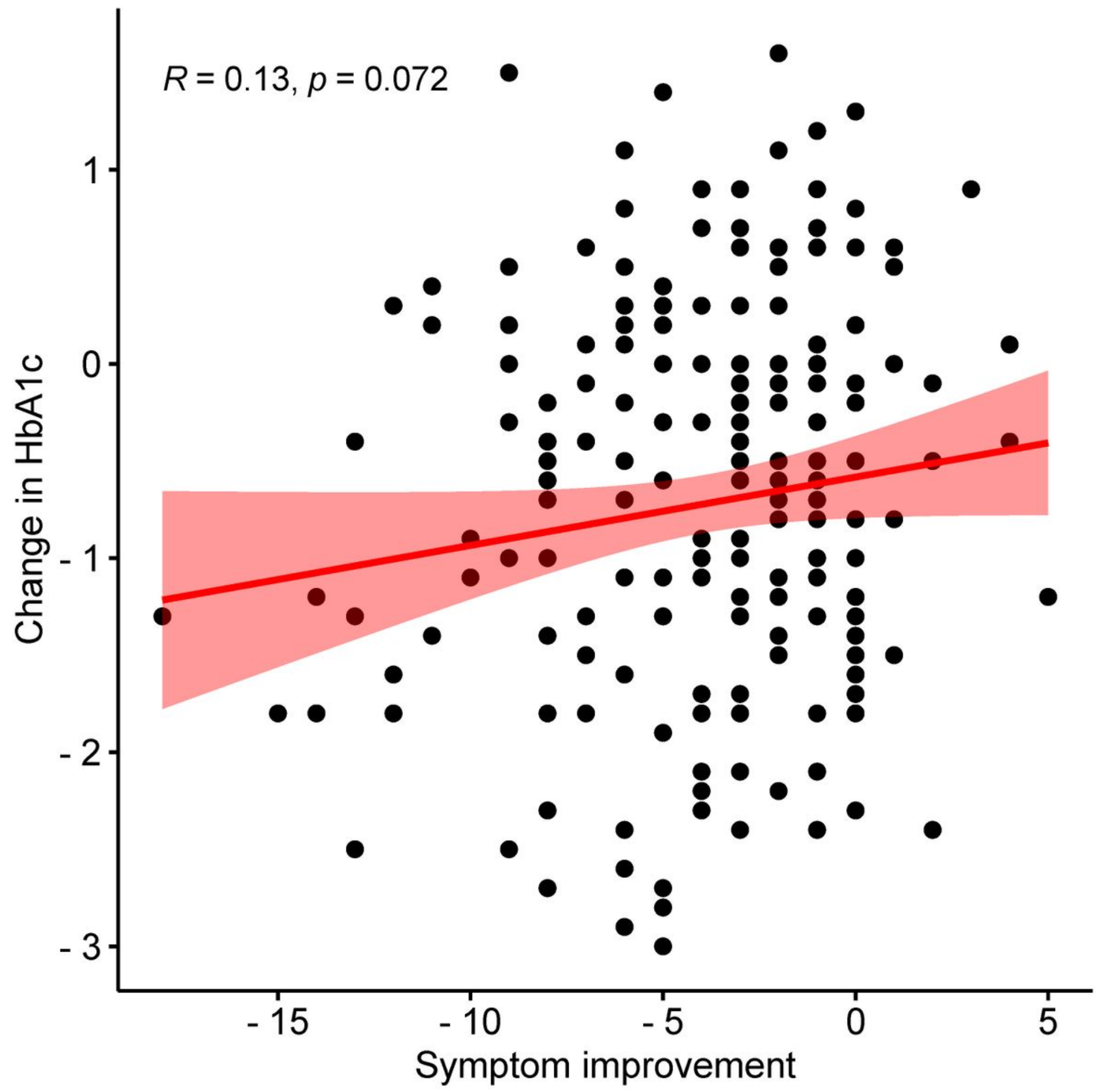

Figure 5

Correlation analysis for symptom improvement and $\mathrm{HbA} 1 \mathrm{c}$ from baseline in patients after receiving a treatment group or control group for 12 weeks 